\title{
Negative Pressure Incisional Therapy and Postoperative Infection after Posterior Approach Primary Total Hip Arthroplasty
}

\author{
Vineet Tyagi ${ }^{1}$, Joseph Kahan ${ }^{2}$, Patrick Huang ${ }^{2}$, Don Li ${ }^{2}$, David Gibson ${ }^{2}$ \\ 1. Orthopaedics, Yale New Haven Hospital, New Haven, USA 2. Orthopaedics and Rehabilitation, Yale University \\ School of Medicine, New Haven, USA
}

Corresponding author: Vineet Tyagi, vineet.tyagi@yale.edu

\begin{abstract}
A major complication after total hip arthroplasty (THA) is infection, which can have devastating clinical and financial results. Silver-impregnated dry dressings, such as Aquacel dressings, and incisional negative pressure dressings (Prevena) have been developed to reduce the rates of surgical site infections (SSIs) after surgery. We retrospectively reviewed the medical records of 235 patients who underwent primary posterior approach THA at our institution during a three-year period. Patients were grouped based on surgical dressing. Rates of SSI were recorded, as well as the effects of factors including age, sex, body mass index, and medical comorbidities. In the high-risk subgroup, defined as BMI $>30$ and ASA $>3$, the infection rate was $2.97 \%$ in the Aquacel group, compared to $1.20 \%$ in the Prevena group. This difference did not reach statistical significance. There was a statistically significant impact on readmissions rate $(p=0.028)$ and reoperation $(p=0.001)$. The findings of this study suggest that negative pressure dressings in carefully selected patients may help to reduce reoperations and readmissions in this subgroup.
\end{abstract}

Received 03/17/2020

Review began 03/18/2020 Review ended 03/19/2020 Published 03/24/2020

\section{(c) Copyright 2020}

Tyagi et al. This is an open access article distributed under the terms of the Creative Commons Attribution License CC-BY 4.0., which permits unrestricted use, distribution, and reproduction in any medium, provided the original author and source are credited.
Categories: Infectious Disease, Orthopedics, Quality Improvement

Keywords: orthopedics, hip, arthroplasty, infection, negative pressure

\section{Introduction}

Total hip arthroplasty (THA) has been shown to be a successful procedure for degenerative changes of the hip, with over $95 \%$ survivorship with 10 years of follow-up [1,2]. As the number of patients with hip osteoarthritis continues to increase, the need for THA is growing in the United States. It is estimated that nearly half a million primary THAs will be performed yearly by 2030 [2].

Infections after total hip arthroplasty can be difficult complications, both in terms of financial effects on the healthcare system and patient morbidity. The incident of prosthetic joint infection after primary THA is estimated to be around 1-2\% [3-5]. Certain medical comorbidities, such as diabetes, rheumatoid arthritis, and obesity, serve as risk factors which predispose patients to infections [6-8]. With an aging population in the United States, these risk factors become more common, and as a result, complication rates after THA are expected to increase $[9-13,14]$.

The financial implications of revision surgeries have been well documented. The cost of revision surgery has significantly increased in the past decade, from approximately $\$ 55,000$ in 2006 to over $\$ 75,000$ by $2013[3,15]$. The cumulative medical cost of revision arthroplasty surgery in the United States is estimated to exceed $\$ 1.62$ billion by $2020[16]$.

Closed incision negative pressure wound therapy (ciNPWT) has been used as a measure to decrease wound drainage, which could potentially help to reduce deep infection [17, 18]. These types of dressings have been historically used for complications, non-healing wounds, or as adjuncts between wound debridement and definitive surgical closure $[19,20]$. Negative pressure dressings function to accelerate the healing process through a variety of mechanisms: increased vascular flow through angiogenesis, decreased edema, and induction of collagen transcription [21-23].

Currently, the use of negative pressure dressings is accepted for the prophylaxis of wound complications in closed surgical wounds in high risk patients. We have previously analyzed the efficacy of negative pressure dressings in primary anterior total hip arthroplasty [18]. There are no specific guidelines that outline indications for the use of incisional negative pressure dressings after primary posterior approach THA. Our goal is to determine preoperatively which patients have a higher risk of developing prolonged wound drainage and subsequent infection, so that we can help surgeons determine who may benefit from incisional negative pressure dressings. We hypothesized that there would be a lower postoperative surgical site infection (SSI) rate in high risk patients who were treated with a negative pressure incisional dressing (Prevena by Acelity / KCI, San Antonio, TX), compared to those with our current "standard" occlusive, antibacterial surgical site dressing (Aquacel by Convatec, Bridgewater, $\mathrm{NJ}$ ). 


\section{Materials And Methods}

\section{Study design}

The goal of this study was to compare infection rates between ciNPWT dressings (Prevena) and a silverimpregnated occlusive island dressing (Aquacel) after primary total hip arthroplasty done through a posterior approach. After receiving Institution Review Board (IRB) approval, we retrospectively reviewed the electronic medical records of 235 patients who underwent primary total hip arthroplasty through a posterior approach at our institution from January 2016 to January 2019.

Inclusion criteria included primary THA through a posterior approach at our institution by a single surgeon (DG). Exclusion criteria included revision hip arthroplasty, postoperative follow-up of less than three months, conversion of a hemiarthroplasty to THA, and age under 18 years. The primary outcome was development of any postoperative SSI. Secondary outcomes included length of stay (LOS), readmission, and need for reoperation. Age, sex, American Society of Anesthesiology (ASA) class, and type of surgical dressing use were determined by reviewing patient medical records. Additional data, including body mass index (BMI), tobacco use, history of autoimmune disease, preoperative International Normalized Ratio (INR), and procedure details, were also obtained directly from patient records.

\section{Surgical technique}

All THAs were performed by a single surgeon who used a posterior approach using the Pinnacle acetabular cup and either a press-fit Summit or S-ROM stem (Depuy Synthes, Warsaw, IN). The surgeon used Aquacel dressings on all of his patients until halfway through the study period (starting July 2018), when he began using Prevena ciNPWT on patients classified as high risk (BMI $\geqslant 30$ and/or ASA class $\geqslant 3$ ) and silverimpregnated island (Aquacel) dressing in the rest of the "standard risk" patients.

\section{Statistical analysis}

Patient and procedure characteristics were tabulated, and differences between the groups were assessed using Pearson's chi-squared test, z score comparison between two proportions, unpaired student t-test between two means, or Fisher's Exact Test to compare patient demographics, ASA class, and procedure types for patients based on dressing category. All calculations were performed using Stata 13.1 (StataCorp, College Station, TX) or Excel (Microsoft, Silicon Valley, CA), and the threshold for significance was a type I error rate of 0.05 adjusted if appropriate for multiple comparisons. A separate subgroup analysis was performed by risk stratifying patients into categorical "standard" and "high risk" patient groups, defined as patients with BMI $\geqslant 30$ and/or ASA class $\geqslant 3$, to determine whether high-risk patients would benefit from iNPWT dressings. Similar analyses were used for the high-risk group including multivariable linear regression modeling.

\section{Results}

Between January 2016 and January 2019, 235 patients met the inclusion/exclusion criteria of our study and underwent a total of 235 total hip arthroplasties via the posterior approach. iNPWT was used in 92 patients and silver-impregnated occlusive dressing was used in 143 patients. Patient demographics and baseline characteristics are demonstrated in Table 1 . There was no significant difference in age, history of diabetes, smoking status, or autoimmune disease between the two groups. Patients who received iNPWT had a higher BMI (39.5) than patients who received an occlusive dressing (29.6), $\mathrm{p}=<0.001$. They also had a mean ASA class of 2.7, compared to 2.5 for the occlusive dressing group, $\mathrm{p}=<0.001$. 


\section{Cureus}

\begin{tabular}{|c|c|c|c|}
\hline Characteristic & Aquacel & Prevena & P-Value \\
\hline Total Patients & 143 & 92 & \\
\hline Average Age & 64.8 & 61.9 & 0.098 \\
\hline$\%$ Male & $42.6 \%$ & $17.4 \%$ & $<0.001$ \\
\hline Average BMI & 29.6 & 39.5 & $<0.001$ \\
\hline Average ASA Class & 2.5 & 2.7 & 0.008 \\
\hline$\%$ High Risk & $70.6 \%$ & $90.2 \%$ & $<0.001$ \\
\hline \% Diabetes Diagnosis & $23.1 \%$ & $26.1 \%$ & 0.601 \\
\hline \% Smoker & $51.0 \%$ & $51.1 \%$ & 0.988 \\
\hline \% Autoimmune Diagnosis & $13.3 \%$ & $21.7 \%$ & 0.092 \\
\hline
\end{tabular}

\section{TABLE 1: Demographics and baseline characteristic data for study groups}

BMI: Body mass index; ASA: American Society of Anesthesiologists.

As shown in Table 2, there was no difference in the rate of SSI between the two groups overall $(\mathrm{p}=0.553)$. The overall SSI rate in this cohort of 235 cases was $1.28 \%$. There was a trend toward lower overall readmission rate among the iNPWT group compared to the occlusive dressing group (6.52\% vs. $10.49 \%)$, but it was not statistically significant $(p=0.43)$. There was a trend toward lower reoperation rates in the iNPWT group, but this was not statistically significant $(\mathrm{p}=0.09)$. There was no difference in administration of tranexamic acid or length of stay between the two groups. Mean operative time of 89 minutes was longer in the Prevena group, $\mathrm{p}=0.001$.

\begin{tabular}{|c|c|c|c|}
\hline Characteristic & Aquacel & Prevena & P-Value \\
\hline Total Patients & 143 & 92 & \\
\hline Surgical Site Infection & $2 \%(n=3)$ & $1 \%(n=1)$ & 0.553 \\
\hline Readmission & $10.49 \%(n=15)$ & $6.52 \%(n=6)$ & 0.430 \\
\hline Reoperation & $10 \%(n=14)$ & $4 \%(n=4)$ & 0.092 \\
\hline \% TXA Administered & $85 \%$ & $91 \%$ & 0.178 \\
\hline Operative Time (minutes) & 72 & 89 & 0.001 \\
\hline Length of Stay (days) & 3.1 & 3.0 & 0.85 \\
\hline
\end{tabular}

\section{TABLE 2: Surgical site infections, outcomes, and operative data between groups}

TXA: Tranexamic acid

In our subgroup analysis of high-risk patients (Tables 3, 4), defined by BMI $\geqslant 30$ and ASA $\geqslant 3$, we found no difference in age, ASA class, history of diabetes, smoking status, or autoimmune diagnosis. Patients in the Prevena subgroup were more likely to be male $(\mathrm{p}<0.001)$ and had a lower BMI of 40.6 , compared to 31.2 in the Aquacel group $(\mathrm{p}<0.001)$. In the high-risk subgroup, the infection rate was $2.97 \%$ in the Aquacel group, compared to $1.20 \%$ in the Prevena group. This difference did not reach statistical significance $(p=0.348)$. There was a lower reoperation rate in the Prevena group ( $13.86 \%$ vs $1.20 \%, \mathrm{p}=0.001)$, as well as readmissions $(14.85 \%$ vs $4.82 \%, p=0.028$ ). The mean operative time was longer in the Prevena group ( 87.2 vs 75 minutes, $\mathrm{p}=0.005$ ). There was no difference in length of stay between the groups. 


\section{Cureus}

\begin{tabular}{|c|c|c|c|}
\hline Characteristic & Aquacel & Prevena & P-Value \\
\hline Total Patients & 101 & 83 & \\
\hline Average Age & 65.5 & 62.1 & 0.064 \\
\hline$\%$ Male & $45 \%$ & $19 \%$ & $<0.001$ \\
\hline Average BMI & 31.2 & 40.6 & $<0.001$ \\
\hline Average ASA Class & 2.8 & 2.8 & 0.817 \\
\hline \% Diabetes Diagnosis & $27 \%$ & $29 \%$ & 0.764 \\
\hline \% Smoker & $53 \%$ & $54 \%$ & 0.893 \\
\hline \% Autoimmune Diagnosis & $16 \%$ & $19 \%$ & 0.594 \\
\hline
\end{tabular}

TABLE 3: Demographics and baseline characteristic data for high-risk subgroup

BMI: Body mass index; ASA: American Society of Anesthesiologists.

\begin{tabular}{|c|c|c|c|}
\hline Characteristic & Aquacel & Prevena & P-Value \\
\hline Total Patients & 101 & 83 & \\
\hline Surgical Site Infection & $2.97 \%(n=3)$ & $1.20 \%(n=1)$ & 0.348 \\
\hline Readmission & $14.85 \%(n=15)$ & $4.82 \%(n=4)$ & 0.028 \\
\hline Reoperation & $13.86 \%(n=14)$ & $1.20 \%(n=1)$ & 0.001 \\
\hline$\%$ TXA Administered & $82 \%$ & $90 \%$ & 0.125 \\
\hline Operative Time (minutes) & 75 & 87.2 & 0.005 \\
\hline Length of Stay (days) & 3.22 & 3.02 & 0.620 \\
\hline TXA: Tranexamic acid & & & \\
\hline
\end{tabular}

Table 5 shows the results of multivariate regression. BMI and ASA class were the two variables $(\mathrm{p}=0.043$ and $\mathrm{p}=0.034$, respectively) associated with surgical site infection in our study. 


\section{Cureus}

\begin{tabular}{|l|l|}
\hline Predictors & P-Value \\
\hline Prevena & 0.024 \\
\hline BMI & 0.043 \\
Age & 0.746 \\
Gender & 0.754 \\
ASA Class & 0.034 \\
Diabetes Mellitus & 0.542 \\
TXA & 0.451 \\
Smoking & 0.531 \\
Autoimmune & 0.099 \\
\hline
\end{tabular}

TABLE 5: Multivariate regression with SSI as dependent variable

BMI: Body mass index; ASA: American Society of Anesthesiologists; SSI: Surgical site infection.

\section{Discussion}

The objective of Prevena iNPWT is to reduce drainage and help decrease the risk of surgical site infections. All infections observed in this study occurred in the high-risk cohort. Our study suggests that the use of Prevena dressings in patients at higher risk of developing infections may help to reduce reoperation and readmissions, and that these patients may benefit from prophylactic use of Prevena dressings.

In patients at risk of developing infection, the use of NPWT has been shown to decrease the incidence of postoperative seroma and hematoma, as well as infection [24]. Research looking specifically at NPWT in orthopaedics is limited, especially in the subset of patients undergoing primary joint arthroplasty. Due to the potential costs associated with negative pressure dressings, understanding their possible benefits in preventing infections after THA is a worthwhile consideration. In the literature, the cited cost of a standard Prevena dressing is $\$ 495$, compared to a standard Aquacel dressing at $\$ 15$ [25, 26]. At our institution in June 2018 , the cost of a Prevena dressing was $\$ 486$, compared to $\$ 39$ for an Aquacel dressing. Therefore, selective use of Prevena dressings is important.

Pachowsky et al. conducted a randomized controlled trial and found that prophylactic negative pressure dressings after primary THA reduced the volume of postoperative seromas [27]. The study did not analyze prosthetic joint infection [27]. Karlakki et al. found that NPWT in primary total hip and knee arthroplasty led to decreased wound exudate and related complications, as well as lower LOS [28]. Patients in the NPWT group also had fewer dressing changes. The study also found that there were fewer dressing changes, as expected, in the NPWT group. No financial analysis was completed, but subgroup analysis found reductions in wound complications for higher risk patients with an ASA $\geqslant 3$ and a BMI $\geqslant 35$ [28]. Siqueira et al. confirmed a literature review and found similar results that NPWT may be preferentially beneficial in highrisk patients. The review identified high risk of bleeding/hemorrhage postoperatively, patient size/weight, and intraoperatively tissue quality as patient factors that may predispose patients to infection after primary THA [29].

Redfern et al. performed a study comparing a historical control group treated with gauze dressings after THA and total knee arthroplasty (TKA) to a prospective cohort of patients treated with NPWT. There was no difference in deep infections between the groups, but there was a lower rate of superficial infections in the NPWT group. The important caveat for this study was a significant difference in baseline characteristics such as gender, surgery site, and medical comorbidities between the two groups. The study also used plain gauze dressings instead of silver-impregnated antimicrobial dressings [30].

One of the primary limitations of this study is its retrospective design, which limits the ability to make broad conclusions. A future prospective randomized controlled study would be needed to further study the effects of dressings on postoperative infections, especially for high-risk patients. The study of a single surgeon's (DG) cases, designed to eliminate variability in patient selection and surgical technique, limits broader applicability. Analysis of a larger patient cohort may have allowed increased data collection and strengthened results. Future randomized studies with more surgeons are needed to better understand the role of NPWT in primary posterior approach THA. 
To our knowledge, this is the first study examining NPWT in a large cohort of primary posterior approach THA. There are no disclosures or conflicts of interest related to either of the dressings used for any of the study authors. Despite the noted limitations of the study, we feel the results reported constitute an important addition to the literature.

\section{Conclusions}

Our findings suggest that the use of incisional NPWT helps to reduce reoperation and readmission rates in high-risk patients after undergoing primary posterior approach THA. There difference in infections did not reach statistical significance, likely due to limitations in study size. Given the significant costs associated with reoperations and readmissions, our data indicates that prophylactic use of iNPWT in high-risk patients may be a useful adjunct to reduce complications. As a result of this data, we have continued to stratify patients and use incisional negative pressure dressings in those patients deemed to have a higher risk of developing infections. Further study is needed regarding the use of incisional NPWT dressings in high risk and revision hip arthroplasty cases.

\section{Additional Information \\ Disclosures}

Human subjects: Consent was obtained by all participants in this study. Yale University Institutional Review Board issued approval 2000022271. The Yale Human Investigation Committee approved this submission following an expedited review. This approval is valid from 2/14/2018 to 2/13/2020 inclusive. Review Comments: This approval is for medical record review only. This approval does not authorize patient contact. The Principal Investigator has attested that each of the qualifying criteria for an extended reapproval period of two years has been met, and that future notification of any changes to the following criteria will be made to the HIC/HSC, and confirmed by the HIC/HSC, prior to enacting such changes. The research study does not include any of the following: • Any funding, including federal training and program project grants and federal no-cost extensions $\bullet$ Subawards issued to Yale where the prime award is federal $\bullet$ FDA regulated components $\bullet$ Sponsor or other contractual restrictions $\bullet$ Clinical interventions (including clinical behavioral interventions) that are greater than minimal risk $\bullet$ Prisoners as subjects $\bullet$ Receipt of an NIH-issued Certificate of Confidentiality to protect identifiable research data. Please be advised that YaleNew Haven Hospital and Yale Medical Group have implemented a new reporting request process. Requests for medical records should be made through JDAT as described at http://medicine.yale.edu/ycci/oncore/availableservices/datarequests/datarequests.aspx. YNHH and Yale University consider it a violation of patient privacy for research personnel to review medical records of patients who have opted out of research use of their records. All record review requests should therefore be through JDAT. The IRB finds that informed consent can be waived for this study per federal regulation 45 CFR 46.116(d). This part of the regulations states that 1) this research involves no more than minimal risk to the subjects, 2) the waiver or alteration will not adversely affect the rights and welfare of the subjects, 3) the research could not practicably be carried out without the waiver and 4) whenever appropriate the subjects will be provided with additional pertinent information after participation. A HIPAA waiver has been approved via expedited review for access to and use of PHI from medical records as described in the approved medical record review protocol without obtaining written approval ("authorization") from the subject for the use of the data. This waiver does not authorize subject contact. The IRB finds that the use or disclosure of protected health information involves no more than a minimal risk to the privacy of individuals, based on (1) an adequate plan to protect the identifiers from improper use and disclosure; (2) an adequate plan to destroy the identifiers at the earliest opportunity consistent with conduct of the research, unless there is a health or research justification for retaining the identifiers or such retention is otherwise required by law; and (3) adequate written assurances that the protected health information will not be reused or disclosed to any other person or entity, except as required by law, for authorized oversight of the research study, or for other research for which the use or disclosure of protected health information would be permitted by this subpart; The IRB also finds that the research could not practicably be conducted without the waiver or alteration; and the research could not practicably be conducted without access to and use or the protected health information. HIPAA regulations require that accounting logs be maintained when researchers access patient records under a waiver of authorization including those approved for recruitment purposes. You are thereby reminded of your obligation to create the log. For further information on maintaining logs and on the accounting of disclosures, please see hipaa.yale.edu. By 12/15/2019, you are to submit documentation for a continuing review. You can request a continuing review by navigating to the active study and clicking Create Modification / CR. Alternatively, you can close the study when the study procedures and the data analysis of identifiable data are fully complete. You can submit a closure request by navigating to the active study and clicking Create Modification/CR. If you wish to change any aspect of this study, such as the study procedures or processes, the informed consent document(s), recruitment activities, or wish to add or remove investigators or study personnel, you must submit a modification to the study. Any changes must be approved by the IRB prior to implementation. Serious, unanticipated, and related adverse events, and unanticipated problems involving risk to subjects or others must be reported generally within 5 days of the PI becoming aware of the event (see Policy 710: Reporting Unanticipated Problems Involving Risks to Subjects or Others, including Adverse Events). In conducting this study, you should refer to and follow the Investigator Manual (HRP-103), which can be found in the IRB Library within the IRB system. Animal subjects: All authors have confirmed that this study did not involve animal subjects or tissue. 
Conflicts of interest: In compliance with the ICMJE uniform disclosure form, all authors declare the following: Payment/services info: All authors have declared that no financial support was received from any organization for the submitted work. Financial relationships: All authors have declared that they have no financial relationships at present or within the previous three years with any organizations that might have an interest in the submitted work. Other relationships: All authors have declared that there are no other relationships or activities that could appear to have influenced the submitted work.

\section{References}

1. Pivec R, Johnson AJ, Mears SC, Mont MA: Hip arthroplasty. Lancet. 2012, 380:1768-1777. 10.1016/S01406736(12)60607-2

2. Kurtz S, Ong K, Lau E, Mowat F, Halpern M: Projections of primary and revision hip and knee arthroplasty in the United States from 2005 to 2030. J Bone Joint Surg Am. 2007, 89:780-785. 10.2106/JBJS.F.00222

3. Bozic KJ, Kurtz SM, Lau E, Ong K, Vail TP, Berry DJ: The epidemiology of revision total hip arthroplasty in the United States. J Bone Joint Surg Am. 2009, 91:128-133. 10.2106/JBJS.H.00155

4. Dale H, Hallan G, Espehaug B, Havelin LI, Engesaeter LB: Increasing risk of revision due to deep infection after hip arthroplasty. Acta Orthop. 2009, 80:639-645. 10.3109/17453670903506658

5. Kurtz SM, Lau E, Schmier J, Ong KL, Zhao K, Parvizi J: Infection burden for hip and knee arthroplasty in the United States. J Arthroplasty. 2008, 23:984-991. 10.1016/j.arth.2007.10.017

6. Baek SH: Identification and preoperative optimization of risk factors to prevent periprosthetic joint infection. World J Orthop. 2014, 5:362-367. 10.5312/wjo.v5.i3.362

7. Bozic KJ, Lau E, Kurtz S, Ong K, Rubash H, Vail TP, Berry DJ: Patient-related risk factors for periprosthetic joint infection and postoperative mortality following total hip arthroplasty in Medicare patients. J Bone Joint Surg Am. 2012, 94:794-800. 10.2106/JBJS.K.00072

8. Phillips JE, Crane TP, Noy M, Elliott TS, Grimer RJ: The incidence of deep prosthetic infections in a specialist orthopaedic hospital: a 15-year prospective survey. J Bone Joint Surg Br. 2006, 88:943-948. 10.1302/0301-620X.88B7.17150

9. Whiting DR, Guariguata L, Weil C, Shaw J: IDF diabetes atlas: global estimates of the prevalence of diabetes for 2011 and 2030. Diabetes Res Clin Pract. 2011, 94:311-321. 10.1016/j.diabres.2011.10.029

10. A Workgroup of the American Association of Hip, Knee Surgeons Evidence Based Committee: Obesity and total joint arthroplasty: a literature based review. J Arthroplasty. 2013, 28:714-721. 10.1016/j.arth.2013.02.011

11. Flegal KM, Carroll MD, Kit BK, Ogden CL: Prevalence of obesity and trends in the distribution of body mass index among US adults, 1999-2010. JAMA. 2012, 307:491-497. 10.1001/jama.2012.39

12. Raikin SM, Kane J, Ciminiello ME: Risk factors for incision-healing complications following total ankle arthroplasty. J Bone Joint Surg Am. 2010, 92:2150-2155. 10.2106/JBJS.I.00870

13. Jones RE: Wound healing in total joint arthroplasty. Orthopedics. 2010, 33:660.

14. Mont MA, Elmallah RK, Cherian JJ, Banerjee S, Kapadia BH: Histopathological evaluation of the anterior cruciate ligament in patients undergoing primary total knee arthroplasty. J Arthroplasty. 2016, 31:284-289. 10.1016/j.arth.2015.07.010

15. Gwam CU, Mistry JB, Mohamed NS, Thomas M, Bigart KC, Mont MA, Delanois RE: Current epidemiology of revision total hip arthroplasty in the United States: national inpatient sample 2009 to 2013. J Arthroplasty. 2017, 32:2088-2092. 10.1016/j.arth.2017.02.046

16. Kurtz SM, Lau E, Watson H, Schmier JK, Parvizi J: Economic burden of periprosthetic joint infection in the United States. J Arthroplasty. 2012, 27:61-65. 10.1016/j.arth.2012.02.022

17. Saleh K, Olson M, Resig S, et al.: Predictors of wound infection in hip and knee joint replacement: results from a 20 year surveillance program. J Orthop Res. 2002, 20:506-515. 10.1016/S0736-0266(01)00153-X

18. Tyagi V, Kahan J, Huang P, et al.: Negative pressure incisional therapy and infection after direct anterior approach primary total hip arthroplasty. Orthopedics. 2019, 42:e539-e544. 10.3928/01477447-20190906-06

19. Stannard JP, Volgas DA, McGwin G, Stewart R, Obremskey W, Moore T, Anglen J: Incisional negative pressure wound therapy after high-risk lower extremity fractures. J Orthop Trauma. 2012, 26:37-42. 10.1097/BOT.0b013e318216b1e5

20. Stannard JP, Volgas DA, Stewart R, McGwin G, Alonso JE: Negative pressure wound therapy after severe open fractures: a prospective randomized study. J Orthop Trauma. 2009, 23:552-557. 10.1097/BOT.0b013e3181a2e2b6

21. Stannard JP, Robinson JT, Anderson ER, McGwin G Jr, Volgas DA, Alonso JE: Negative pressure wound therapy to treat hematomas and surgical incisions following high-energy trauma. J Trauma. 2006, 60:13011306. 10.1097/01.ta.0000195996.73186.2e

22. Stannard JP, Singanamala N, Volgas DA: Fix and flap in the era of vacuum suction devices: what do we know in terms of evidence based medicine?. Injury. 2010, 41:780-786. 10.1016/j.injury.2009.08.011

23. Moues CM, van den Bemd GJ, Heule F, Hovius SE: Comparing conventional gauze therapy to vacuumassisted closure wound therapy: a prospective randomised trial. J Plast Reconstr Aesthet Surg. 2007, 60:672681. 10.1016/j.bjps.2006.01.041

24. Grauhan O, Navasardyan A, Hofmann M, Muller P, Stein J, Hetzer R: Prevention of poststernotomy wound infections in obese patients by negative pressure wound therapy. J Thorac Cardiovasc Surg. 2013, 145:13871392. 10.1016/j.jtcvs.2012.09.040

25. Kuo FC, Chen B, Lee MS, Yen SH, Wang JW: AQUACEL(R) Ag surgical dressing reduces surgical site infection and improves patient satisfaction in minimally invasive total knee arthroplasty: a prospective, randomized, controlled study. Biomed Res Int. 2017, 2017:8. 10.1155/2017/1262108

26. Matatov T, Reddy KN, Doucet LD, Zhao CX, Zhang WW: Experience with a new negative pressure incision management system in prevention of groin wound infection in vascular surgery patients. J Vasc Surg. 2013, 57:791-795. 10.1016/j.jvs.2012.09.037

27. Pachowsky M, Gusinde J, Klein A, et al.: Negative pressure wound therapy to prevent seromas and treat surgical incisions after total hip arthroplasty. Int Orthop. 2012, 36:719-722. 10.1007/s00264-011-1321-8 


\section{Cureus}

28. Karlakki SL, Hamad AK, Whittall C, Graham NM, Banerjee RD, Kuiper JH: Incisional negative pressure wound therapy dressings (iNPWTd) in routine primary hip and knee arthroplasties: a randomised controlled trial. Bone Joint Res. 2016, 5:328-337. 10.1302/2046-3758.58.BJR-2016-0022.R1

29. Siqueira MB, Ramanathan D, Klika AK, Higuera CA, Barsoum WK: Role of negative pressure wound therapy in total hip and knee arthroplasty. World J Orthop. 2016, 7:30-37. 10.5312/wjo.v7.i1.30

30. Redfern RE, Cameron-Ruetz C, O'Drobinak SK, Chen JT, Beer KJ: Closed incision negative pressure therapy effects on postoperative infection and surgical site complication after total hip and knee arthroplasty. J Arthroplasty. 2017, 32:3333-3339. 10.1016/j.arth.2017.06.019 